\title{
Correction to: Weed Detection Approach Using Feature Extraction and KNN Classification
}

Gurpreet Khurana and Navneet Kaur Bawa

Correction to:

Chapter "Weed Detection Approach Using Feature Extraction and KNN Classification" in:

V. C. Pandey et al. (eds.), Advances in Electromechanical Technologies, Lecture Notes in Mechanical Engineering, https://doi.org/10.1007/978-981-15-5463-6_60

In the original version of the book, the following belated correction has been incorporated.

The reference citation in figure 1 and figure 2 has been changed as follows.

From: Fig. 1 Steps for weed detection [6]

Fig. 2 Weed image [9]

To: Fig. 1 Steps for weed detection [10]

Fig. 2 Weed image [10]

The chapter and book have been updated with the changes.

The updated version of this chapter can be found at

https://doi.org/10.1007/978-981-15-5463-6_60 